\title{
Runtime Management of Multi-level SLAs for Transport and Logistics Services
}

\author{
Clarissa Cassales Marquezan ${ }^{1}$, Andreas Metzger $^{1}$, Rod Franklin $^{2}$, and Klaus Pohl ${ }^{1}$ \\ 1 paluno (The Ruhr Institute for Software Technology) \\ University of Duisburg-Essen, Essen, Germany \\ \{clarissa.marquezan, andreas.metzger, klaus.pohl\}@paluno.uni-due.de \\ 2 Kühne Logistics University, Hamburg, Germany and \\ Kühne + Nagel Management AG, Schindellegi, Switzerland \\ rod.franklin@the-klu.org
}

\begin{abstract}
SLA management of non-computational services, such as transport and logistics services, may differ from SLA management of computational services, such as cloud or web services. As an important difference, SLA management for transport and logistics services has to consider so called frame SLAs. A frame SLA is a general agreement that constitutes a long-term contract between parties. The terms and conditions of the frame SLA become the governing terms and conditions for all specific SLAs established under such a frame SLA. Not considering the relationships between frame SLAs, specific SLAs and QoS monitoring information may lead to partial conclusions and decisions, thereby resulting in avoidable penalties. Based on a real industry case in the transport and logistics domain, this paper elaborates on a multi-level run-time SLA management approach for non-computational services that takes into account those relationships. We describe a cloud-based software component, the BizSLAM App, able to automatically manage multi-level SLAs by extending SLA management solutions from service-oriented computing. We demonstrate the feasibility and usefulness of the SLA management approach in an industrial context.
\end{abstract}

\section{Introduction}

Managing Service Level Agreements (SLAs) is an essential task for all kinds of services, be they computational (e.g., cloud services or web services) or non-computational (such as transport and logistics, manufacturing, energy, or agriculture services). The major goals of SLA management are to monitor, check, and ensure that the expected QoS attributes are met during service execution. Expected QoS attributes are expressed in terms of Service Level Objectives (SLOs) that are part of SLAs. In the computational domain, SLA management has been extensively researched. A diversity of languages and tools have been developed [24|2|15]16 19].

SLA management for transport and logistics services is just beginning to be investigated [12]. This especially holds true for automating SLA management, which is fostered by the increasing digitization of SLAs of transport and logistics services together with the need to share SLA information among the participants in a business process. The transport and logistics domain thus significantly would benefit from the techniques

X. Franch et al. (Eds.): ICSOC 2014, LNCS 8831, pp. 560 5742014.

(c) Springer-Verlag Berlin Heidelberg 2014 
and methods developed by the services community for computational services. This paper investigates opportunities for extending techniques developed for computational services to non-computational services in the transport and logistics domain by starting from an understanding of industry requirements and their potential for automation.

Traditionally, managing computational SLAs involves handling two levels of information: (1) QoS monitoring data collected during service execution used, for instance, to check whether the service-level objectives are met; and (2) the actual SLAs that specify expected and agreed to service-level objectives.

SLA management for transport and logistics requires an additional level of information: (3) terms and conditions of so-called frame SLAs. A frame SLA is a general agreement that constitutes a long-term agreement (e.g., one year) between parties that have decided to work together. During this period of time, each request for service execution creates a specific SLA (which is equivalent to the SLA at level (2) for computational services). The terms and conditions of the frame SLA become the governing terms and conditions for all specific SLAs established under the frame SLA. In contrast to computational services, the frame SLA is the actual legally binding document between the two partners. The advantage of frame SLAs is that they simplify the execution of services that will be delivered in a repeated manner over an extended time frame. These services can all be executed under the same agreement without having to renegotiate SLAs and SLOs for each service execution.

To automate the SLA management process for transport and logistics services executed under frame agreements requires dedicated solutions capable of handling these three levels of information at run-time in an automated fashion. It is important to consider the multi-level relationships between frame SLA, specific SLA, and actual QoS measurements. Otherwise, SLA management may lead to wrong conclusions and decisions that service levels have or have not been met, resulting in inapplicable and avoidable penalties. Section 2 elaborates on these problems using industry data, thereby motivating the industry needs for such automated solutions.

In our previous work [12], we presented an analyzer component for runtime SLA management of transport and logistics services, providing a computational solution for automatic SLA checking at run-time. In this paper, we integrate this analyzer component into the BizSLAM App, which is developed on top of FIspace.eu, a cloud-based business collaboration platform [23]. To this end, we $(i)$ define an extensive data model for transport and logistics services and (ii) implement dedicated user interfaces for managing SLAs. Section 3 introduces the conceptual foundations and features of app as well as the data model used to express and relate the multiple levels of SLA information. It also describes how our SLA management approach advances the state of the art.

Section 4 discusses feasibility and usefulness of our SLA management approach, applying the BizSLAM App prototype to a specific scenario in SLA management.

\section{Problem Statement and Industry Needs}

Transport and logistics services can account for between $10 \%$ to $20 \%$ of a country's Gross Domestic Product, and $\mathrm{CO}_{2}$ emissions from transport activities amount to $14 \%$ of total greenhouse gas emissions. Therefore, an improvement in how efficiently these 
services are provided can dramatically increase competitiveness and sustainability. Evidence suggests that improved management of transport processes through advanced IT could yield efficiency gains in the range from 10\% to 15\% [1]. Many opportunities for employing IT to optimize and improve transport and logistics processes can be listed, such as better business collaboration support [23], real-time information handling, better transport and logistics planning tools, predictive process management [17], and enhanced SLA management solutions [9].

In this paper, we focus on enhanced SLA management. More specifically, we look at transport and logistics service level agreements (or SLAs) and their management during the execution of transport and logistics processes. Illustrated by concrete examples from an industry dataset (available from http://www.s-cube-network.eu/c2k), we elaborate on the current situation in industry and the key business requirements for enhanced IT solutions for SLA management in this domain. The industry dataset is based on Cargo 2000 logs covering five months of business operations. Cargo 2000 is a standard established by IATA, the International Air Transport Association, enabling the cross-organizational monitoring of transport processes.

Figure 1 shows the relationship between the actual and planned units of cargo associated with the transportation processes covered by this dataset. The "planned" axis denotes the number of units the logistics service client booked and thus constitutes the number of units that the logistics service provider was expecting to receive from the client. This booked value thus forms part of an SLA between the logistics service client and the logistics service provider. The "actual" axis indicates the effective cargo units received by the logistics service provider at the beginning of the air transport service.

From the perspective of the logistics service provider, all the circles off the diagonal line in Figure 1 would theoretically indicate SLA violations since the actual amount delivered by the customer does not comply with what has been booked. However, the aforementioned information is not sufficient to determine whether an actual SLA

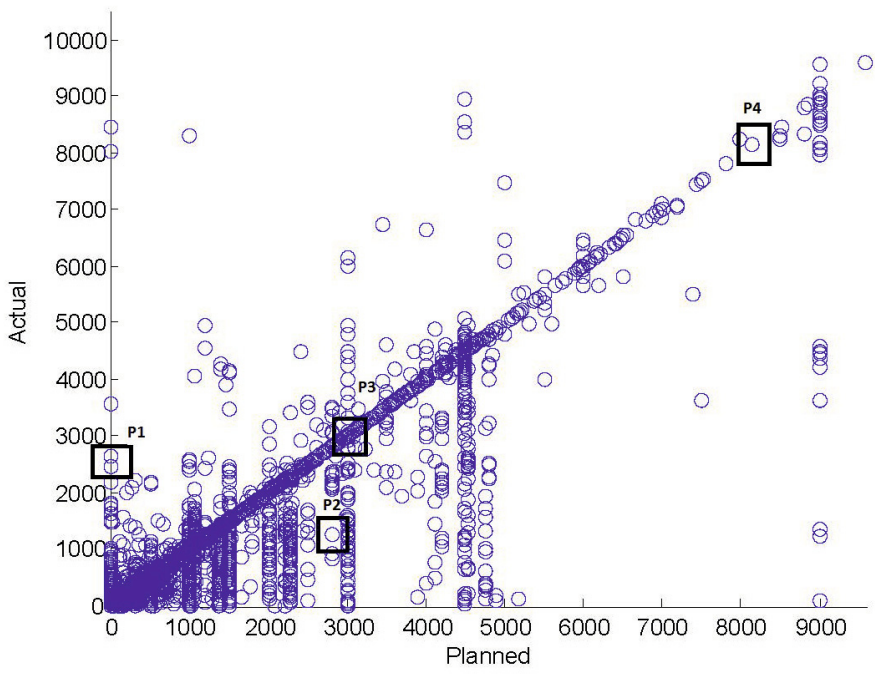

Fig. 1. Planned vs. actual weight of cargo for real-world transport processes (adapted from [8]) 
violation happened for the transport and logistics service. As discussed in Section 1 , the relationships of the three levels of information must be considered, i.e., how the actual measured QoS value, the planned value of the specific SLA, and the frame SLA are related. For instance, assume that the points highlighted by the boxes in Figure 1 are associated with the same frame SLA. Also, assume that this frame SLA establishes that a logistics service client may ship up to 25 containers, each with up to 3000 units of cargo, within the time span of one year. This means that whenever the logistic service client delivers a container with up to 3000 units to be transported, this delivery complies with the frame SLA and the cargo should be transported for the fixed price established under the frame SLA (provided that the number of containers delivered previously does not exceed the established limit of 25). Above this threshold, the fixed price might not apply and may thus require re-negotiating the SLAs.

The boxes P1, P2, P3, and P4 in Figure 1 show the actual amount delivered by the logistics service client (axis Y) versus the planned and reserved amount of cargo to be transported by the logistics service provider (axis X). An analysis of these points without factoring in the frame SLA would indicate that points P3 $(3000,3000)$ and P4 $(8000,8000)$ do not constitute SLA violations, while P1 $(0,2900)$ and P2 $(2900,1200)$ constitute SLA violations. In this case, penalties should be applied for the service execution of points P1 and P2. Now, taking into account the frame SLA, we actually reach a different conclusion: We find that points P1, P2, and P3 do not constitute violations since the respective amount of cargo in these service executions is under, or equal to, the amount established in the frame SLA (i.e., 3000 units of cargo). In contrast, the service execution represented by point $\mathrm{P} 4$ does constitute a violation of the frame SLA.

Currently, industry follows a manual process to check whether the SLOs of the specific agreements (i.e., each individual service execution) conform with the SLOs of the related frame agreement. The numbers provided by a large company from the transport and logistics domain show that in a given month up to 100,000 transports may have to be handled by the logistics service provider [17]. Each of these transports may be associated with a specific agreement, meaning that the number of specific agreements to be checked by a large transport and logistics company could reach up to hundreds of thousands of documents per month. This clearly requires automated support.

The situation faced by industry today, and as presented above, is mainly caused by the following limitations: First, frame SLA information is currently not available in real-time to the down-stream individuals in charge of the actual operations of the logistics service providers. Second, there are currently no standards for representing SLAs in the domain in a structured way. Third, as a consequence from the aforementioned limitations, the SLA management in the transport and logistics domain is manually performed in a "post-morten" fashion (i.e., long after the execution of the service). The remainder of this paper introduces our solution to address these limitations.

\section{The BizSLAM App}

This section introduces the BizSLAM App, a protoype implementation of a multi-level SLA management component. We first provide the conceptual foundations for multilevel SLAs (Section 3.1) and then introduce the key features of the BizSLAM App 
(Section 3.2). One key element of the solution is an extensive data model that includes the major data types found in SLAs for transport and logistics services (Section 3.3). The section concludes with a discussion of related work (Section 3.4).

\subsection{Specifics of Transport and Logistics SLAs}

Figure 2 depicts the main concepts of transport and logistics SLAs. Each agreement consists of three fundamental aspects. First, the association among the Logistics Service Provider and the Logistics Service Client as illustrated at the top of Figure 2. Second, $S L O s$ that define the expected quality attributes of a transport and logistics service. Third, a set of Terms and Conditions including liability and penalty terms that become applicable once a deviation from the SLOs is identified.

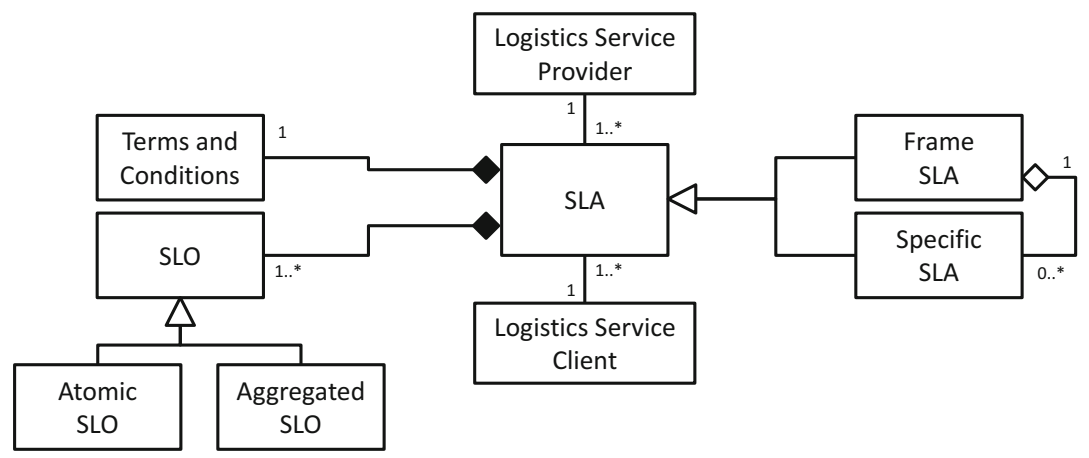

Fig. 2. UML model representing key concepts of Transport and Logistics SLAs

Following from our observations in Section 2, an SLA can either be a Frame SLA or a Specific SLA. Each Specific SLA is related to exactly one Frame SLA. This also leads to two types of SLOs specified in the domain: An Atomic SLO defined in a frame SLA specifies a quality guarantee that has to be met by each of the specific SLAs. In our example from Section 2, the maximum of 3000 cargo units constitutes such an atomic SLO. Each specific SLA established under the related frame SLA may only define a maximum of 3000 cargo units. Another example of an atomic SLO is transit time, defining a maximum time span during which each individual transport must occur. In contrast, an Aggregated SLO defined in a frame SLA specifies a quality guarantee in terms of an accumulative value based on the respective SLOs in the specific SLAs. In our example from Section 2, the maximum number of 25 containers per year constitutes such an aggregated SLO. This means that the sum of all containers defined in the specific SLAs may not be more than 25. The two types of SLAs (frame and specific) together with the two types of SLOs (atomic and aggregated) constitute the core for supporting runtime and automated SLA management for transport and logistics services.

\subsection{Features of the App}

The main purpose of the BizSLAM App is to make SLOs from frame SLAs and specific SLAs available during run-time, thereby fostering conformance and consistency checks. 
The BizSLAM App is developed on top of FIspace.eu, a cloud-based business-tobusiness collaboration platform [23 17]. The app consists of a front-end and back-end. The front-end provides a graphical user interface and is realized as a $\mathrm{W} 3 \mathrm{C}$ widget using HTML, CSS and JavaScript. The back-end is implemented in Java and employs the Spring framework to provide REST APIs for connection with the front-end.

The core capabilities of the BizSLAM App are (1) online access to SLA information for all participants while respecting privacy and security requirements (SLA Operations), and (2) real-time detection and signaling of SLA violations (SLA Analytics).

As part of SLA Operations, the BizSLAM App provides support for storing, reading, deleting, updating, and searching for SLA data. The SLA data stored in the BizSLAM App is a subset of the legal contract agreed to by the transport and logistics partners. This subset contains the data (specifically SLOs) to drive the daily activities of transport and logistics service execution. It is out of the scope of the BizSLAM App to engage in the actual contracting negotiation and agreement. Instead, the focus lies on making the agreed SLOs available to participants during runtime. The relevant SLA data is stored in the form of Linked-USDL documents. To this end, the BizSLAM App employs an open source, reusable software component 1 . Details of the data model used for storing SLA data are discussed in Section 3.3 .

As part of SLA Analytics, the BizSLAM App provides services for an automatic analysis of effective as well as potential SLA violations at runtime. Examples include the detection of repetitive violations of the agreed SLOs together with recommendations for changing the terms of the SLA, checking at a very early stage of the transport and logistics service planning process if the SLOs of a specific SLA comply with the SLOs of the frame SLA established between the parties, as well as proactive notification about opportunities to establish or modify SLAs.

One core element of the BizSLAM App is an analyzer component for automated compliance checks of specific SLAs and frame SLAs. The details of the analyzer component have been presented in our previous work [12]. Basically, the analyzer component translates SLAs into a Constraint Satisfaction Problem (CSP), as agreement terms can be naturally expressed as constraints over a service domain.

\subsection{SLA Data Model}

Currently, there is no "de facto" standard in the transport and logistics domain that is able to represent different types of SLAs and the diversity of SLOs. Therefore, based on experience gathered from interviews and repeated interactions with transport and logistics partners from industry, we defined an extensive data model for SLAs in that domain. As a result, the data model consolidates all information relevant for SLA management of transport and logistics services. Nowadays, such information is scattered across e-mails, spread sheets, and paper documents.

The data model defines all information constituting a transport and logistics SLA, called Transport and Logistics SLA Vocabulary. This model allows for the customization of the SLA and SLO types to meet the specific requirements of different sectors and modes of operations in the industry. Primarily, the data model supports the process of

${ }^{1}$ http://catalogue.fi-ware.org/enablers/repository-sap-ri 
introducing SLA information during the execution of services. The data model thereby provides a common frame for expressing SLAs. Based on such a common frame, contract terms (and their definitions) can be announced by the logistics service provider and agreed on by the logistics service users, thereby ensuring "semantic" equivalence of the SLOs employed in the various SLAs (e.g., see Section 6 in [21]).

The design of our data model builds upon initial data models proposed by the EU e-Freight project [7]. It is implemented in Linked-USDL, which is a version of USDL (the Unified Service Description Language that builds upon the Linked Data principles and the Web of Data. To this end, we define our Transport and Logistics SLA Vocabulary as an RDF vocabulary, which is depicted in Figures 3 , Concepts in green and purple indicate the extensions we introduced on top of the e-Freight model. Purple concepts represent transport and logistics concepts defined in existing data models. Blue concepts represent existing vocabularies adopted by Linked-USDL, such as GoodRelations $\sqrt{3}$ and vCard $\sqrt{4}$. Due to space limitations we focus the following description on the most important concepts of the data model.

Part A includes the basic concepts for the Transport and Logistics SLA Vocabulary. The central concept is Contract, which links to all other concepts in the vocabulary (as explained below). Contract holds the information about the established SLA like issue date, issue time, validity period, involved parties and so forth. In order to differentiate between frame and specific SLAs, the ContractType concept is used. The links between

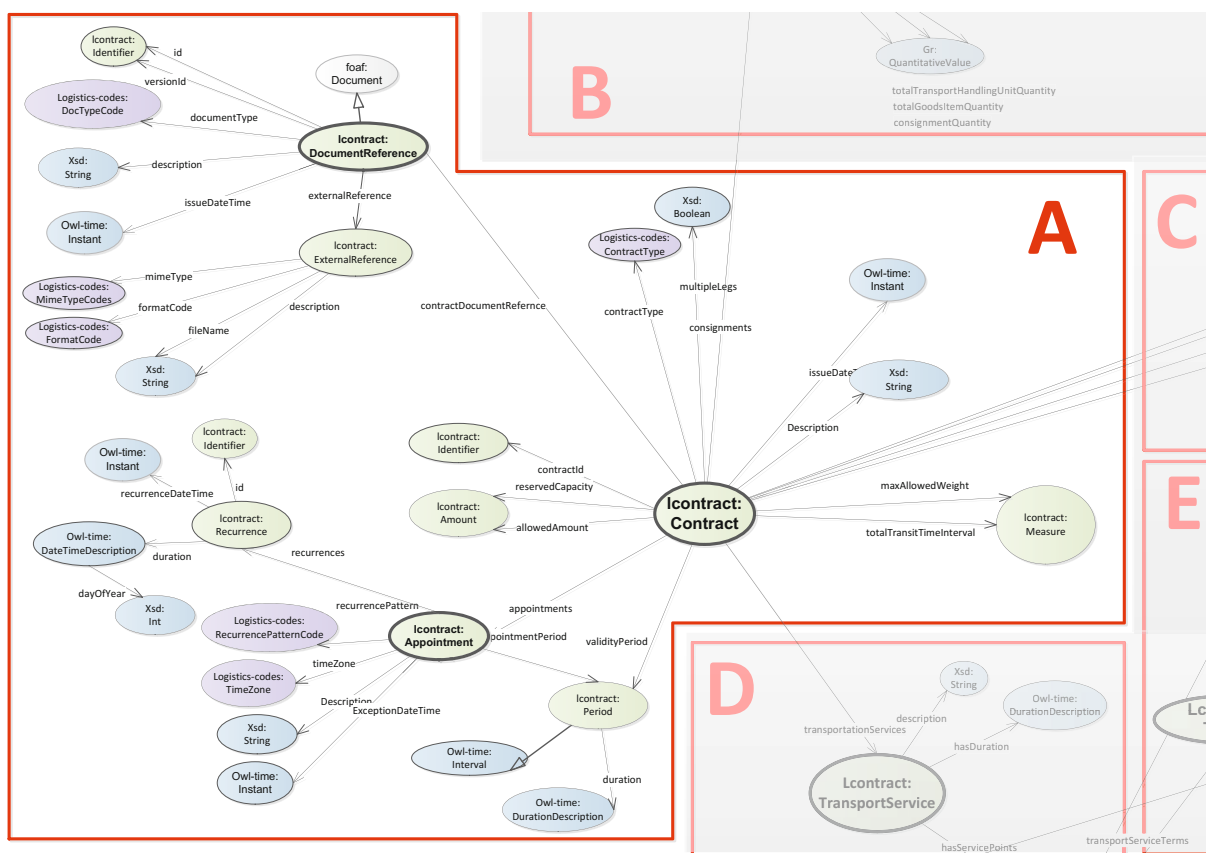

Fig. 3. Data model for Transport and Logistics SLAs represented as RDF graph (Part A)

\footnotetext{
2 http://linked-usdl.org/

3 http://www.heppnetz.de/ontologies/goodrelations/v1

4 http://www .w3.org/Submission/vcard-rdf/
} 


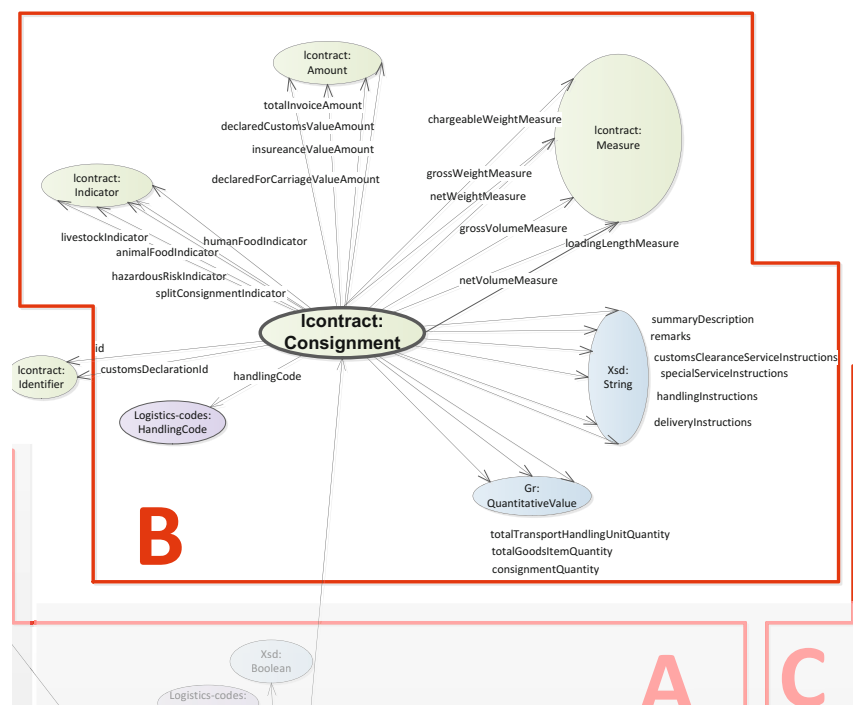

Fig. 4. Data model for Transport and Logistics SLAs represented as RDF graph (Part B)

frame SLA and specific SLAs are realized by means of the ServicePoint concept introduced in Part E of the data model.

Part B is designed to enable a very detailed description of the goods that could be transported under the SLA terms. Nonetheless, the attributes and relationships of this section of the SLA are not mandatory and can be used according to the needs of partners establishing the SLA. Examples of concepts that allow for expressing detailed

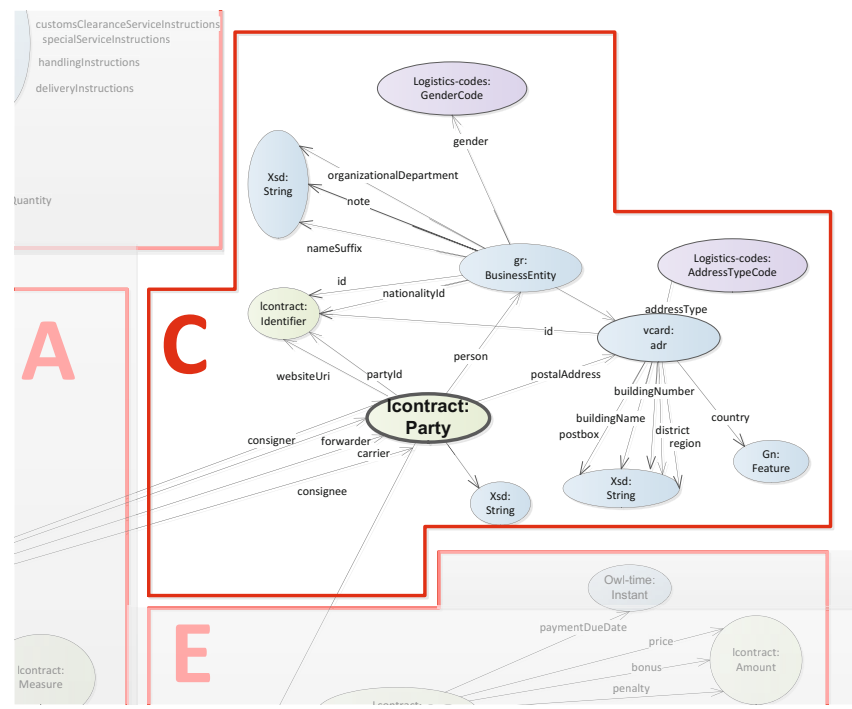

Fig. 5. Data model for Transport and Logistics SLAs represented as RDF graph (Part C) 


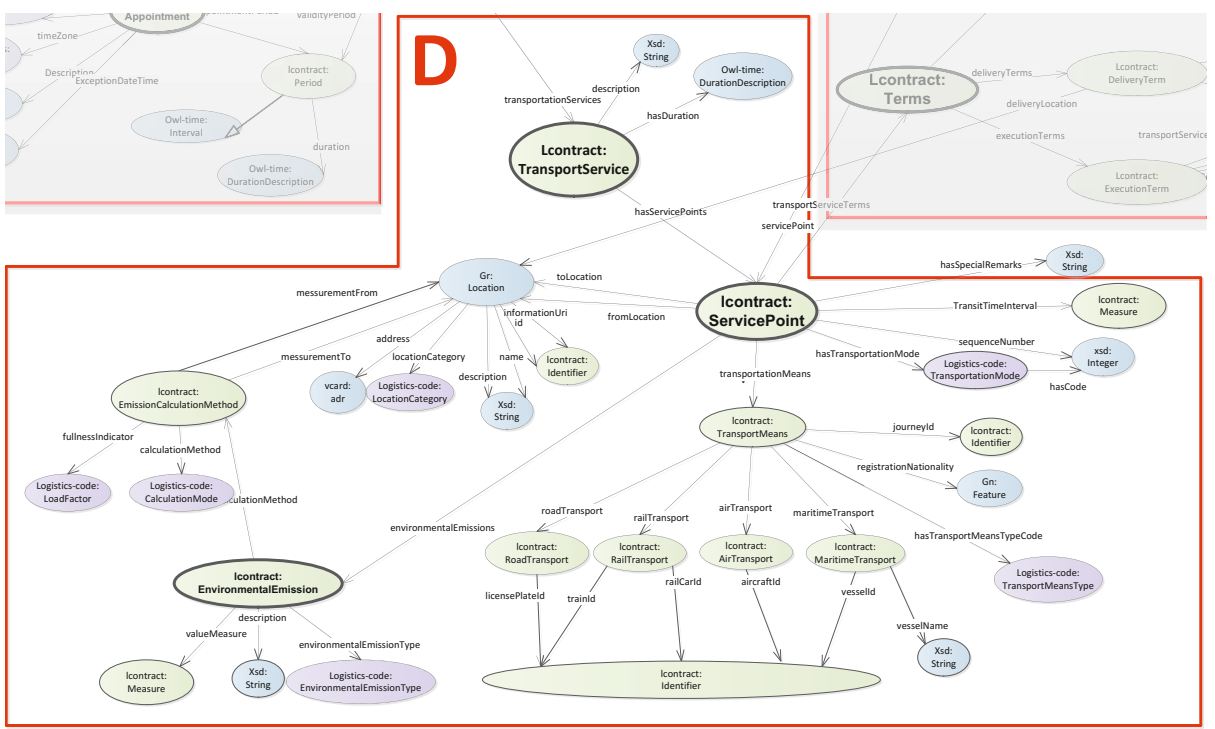

Fig. 6. Data model for Transport and Logistics SLAs represented as RDF graph (Part D)

information of goods include Measure (e.g., volume, weight), Amount (e.g., amount declared for customs), and Indicators (e.g., hazardous).

Part C describes the parties associated with an SLA. The Party concept and its associated concept defines the information about the provider and consumer of the agreed contract.

Part D depicts the transportation service agreed among the parties of the SLA. The concepts Transport Service and Service Point are the most relevant in this part of the vocabulary. The ServicePoint concept is used to specify a single transportation service (transport leg) with a specific sequence number (also see Part E). We designed the Transport and Logistics SLA Vocabulary in such a way that two basic representations

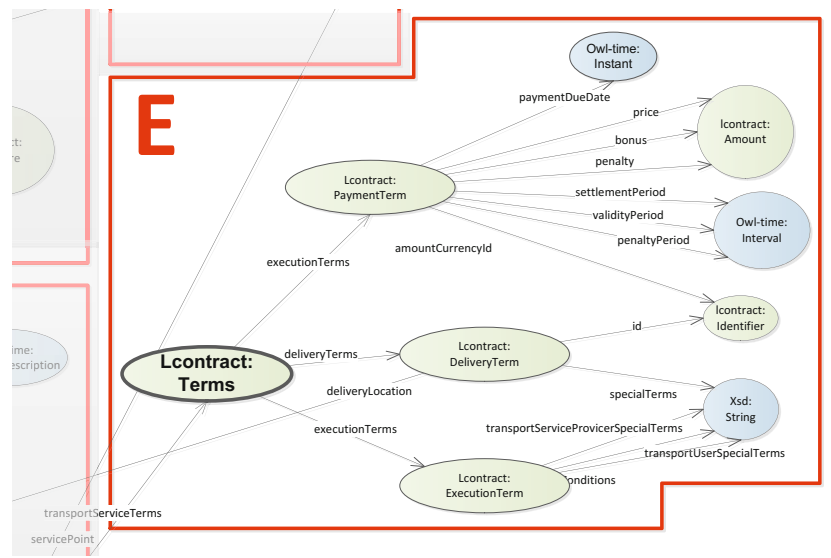

Fig. 7. Data model for Transport and Logistics SLAs represented as RDF graph (Part E) 
of transport and logistics SLAs can be chosen depending on the actual situation faced in practice: The first representation uses only one service point to define a transport and logistics SLA. This means that the SLA specifies SLOs for a single transportation leg. The granularity of this leg is irrelevant. For example, the leg could be from Turkey to UK, or from the airport of Amsterdam to the port of Rotterdam. The second representation uses multiple service points, each with individual SLOs. In this case, the vocabulary is able - in a more fine-grained way - to represent SLAs that specify different SLOs for each transportation service. For example, if the SLA specifies that goods from partner P1 should be transported by partner P2 from Turkey to the UK, this may involve two service points with specific SLOs: one for sea transportation from Turkey (i.e., the first leg of transportation service), and a second for road transportation once the goods have arrived in the UK (i.e., the second leg).

Part E associates the terms of the SLA to each Service Point. For each of the service points certain terms must be defined. Using the previous data models as a basis, we defined three minimal terms that must be specified for each service point using the contract vocabulary: payment, delivery, and execution. The ServicePoint concept can thereby be used to define multi-leg, multi-party, as well as multi-level SLAs.

\subsection{Existing Solutions and Related Work}

Formalization of contracts and automatic conformance checking of contracts has received considerable interest from a wide variety of research areas since the 1980ies [13]. Work includes approaches for automatic monitoring of formalized contracts [25|13] and for managing multi-party contracts [18]. Recently, contract management has received attention for the management of SLAs associated with web services and service-based applications [22 10 15], as well as cloud services [14 5|20 16]. Approaches that use frameworks such as the ones provided by SLA@SOI [24] and WS-Agreement [192] are also available. In summary, all these aforementioned approaches fail to consider frame SLAs and thus the relationships between the three levels of information needed for managing transport and logistics SLAs.

Considering domain-specific approaches for SLA management in transport and logistics many of those efforts rely on Service Oriented Computing principles and techniques, such as the ones presented in [4|27]. They thus also share the aforementioned shortcomings. Complementary efforts have addressed the goal of measuring KPIs among partners in the supply chain. One class of approaches relies on the definition and analysis of contract models for a multi-party collaborative business process, so-called 3PL (3rd Party Logisitcs) or 4PL services [28]. These types of business processes result in a supply chain with collaborative tasks executed by different logistics partners. However, the approach does not provide facilities for runtime SLA management. A different approach developed for transport and logistics services includes a platform based on a service-oriented approach for managing contracts in 4PL businesses [3]. The proposed platform is primarily focused on coordinating business processes among the different partners but not targeted at managing the SLAs among the partners. Finally, different ontology representations of transport and logistics services have been proposed [6]11|26]. They focus on representing services offered by a logistics service provider, match-making for such services and mediation among terms of 
different information models used by different logistics partners. Yet, they do not focus on representing SLA information once an agreement has been established.

In our previous work [12], we presented a first solution for the run-time management of multi-level transport and logistics services. Specifically, we introduced a computational solution for automatic SLA checking at run-time that employed WS-Agreement to formally represent frame and specific SLAs, and that used CSP solvers to check for inconsistencies. In this paper, we integrate this technical approach into an overall systems perspective and provide evidence for the industrial relevance, applicability and usefulness of such an approach in the transport and logistics domain.

\section{Feasibility and Usefulness}

This section demonstrates the feasibility of the BizSLAM App (Section 4.1) and discusses the usefulness of applying the App in an industrial context (Section 4.2).

\subsection{Feasibility}

As described above, the BizSLAM App can be applied to automatically determine inconsistencies in multi-level SLAs during business operations. Figure 3.4 depicts a realworld scenario that shows typical inconsistencies that can be detected. In the given scenario, a logistics service client has established a frame SLA A with a logistics service

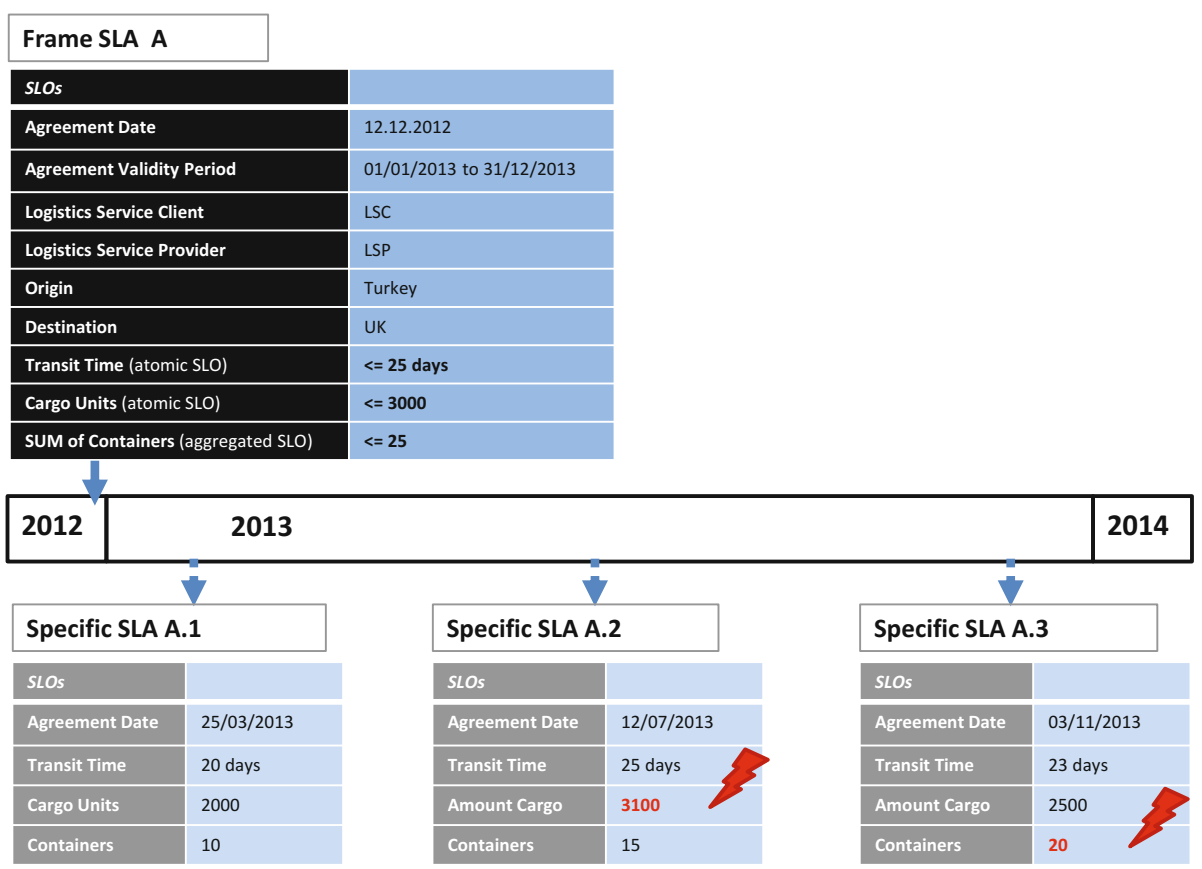

Fig. 8. Inconsistencies between frame and specific agreements 
provider. This scenario defines two atomic SLOs as part of the frame SLA: a maximum of 25 days Transit Time as well as a maximum of 3000 Cargo Units. In addition, the frame SLA defines an aggregated SLO that defines 25 as the SUM of Containers to be transported during the validity period of the frame SLA. For each execution of a transport and logistics service under the frame SLA A, a specific SLA is created. Figure 3.4 shows three such specific SLAs: A.1, A.2 and A.3.

In the scenario depicted in Figure 3.4, two violations occur that are detected by the BizSLAM App as shown in Figure 4.1 The automated conformance check of the BizSLAM App detects these violations immediately, i.e., as soon as they occur, and issues so called pre-violation alerts (the red boxes in Figure 4.1). These alerts inform the logistics service users that if they insist on the chosen SLOs (e.g., in order to ensure timely delivery of goods) this might imply penalties for violating the frame SLA at the end of the validity period of the frame SLA.

\section{Creation of Specific SLA A.2}

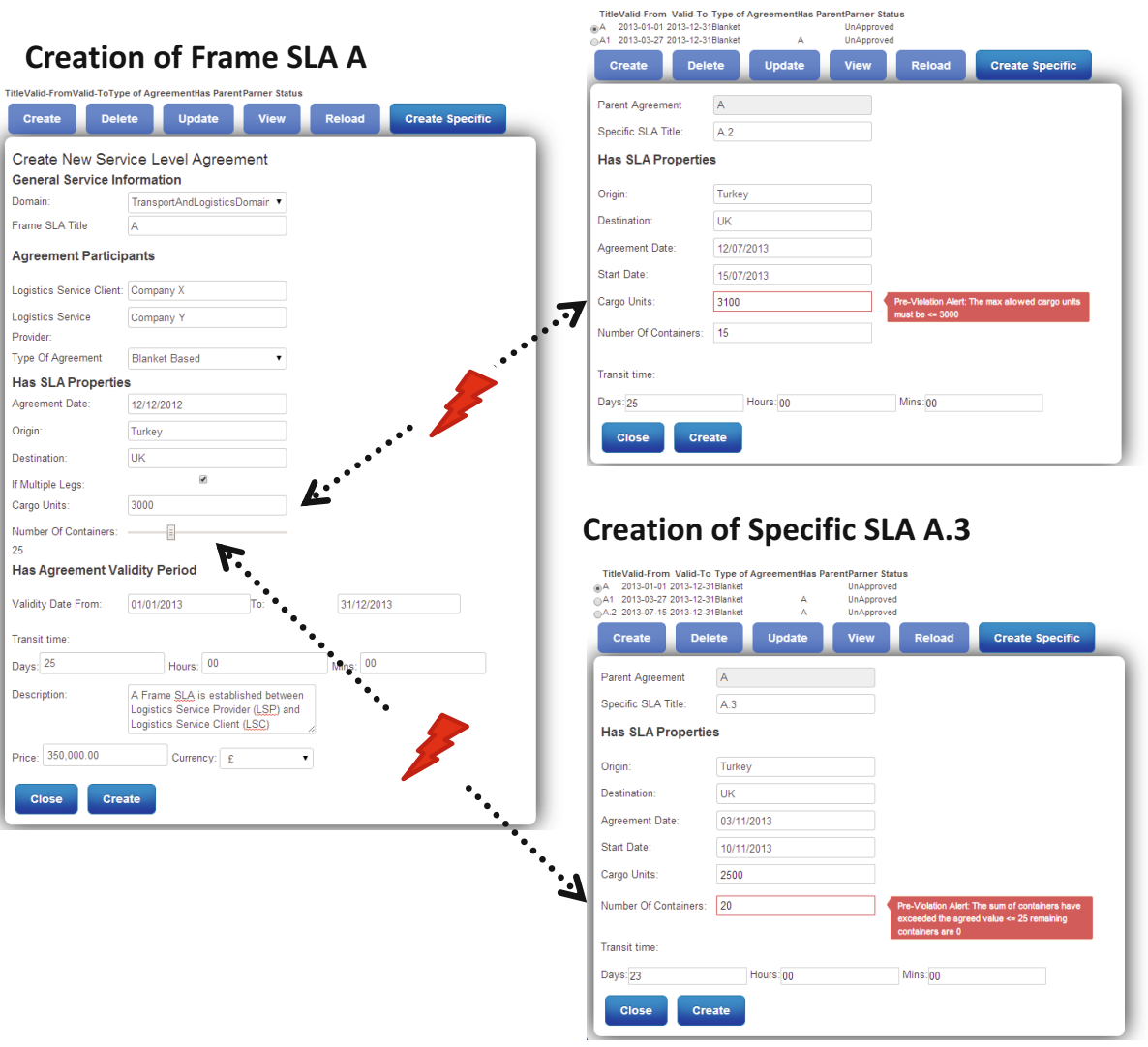

Fig. 9. BizSLAM App detecting inconsistencies between specific and frame agreements 
Violation 1: According to the frame SLA, only 3000 cargo units may be transported for each specific SLA. However, the specific SLA A.2 asks for a cargo volume of 3100 and thus violates the atomic SLO Cargo Units specified in the frame SLA.

Violation 2: A total of 25 containers may be contracted during the validity period of the frame SLA. When the specific SLA A.3 asks for 20 containers, 25 containers have already been contracted in the previous specific SLAs A.1 and A.2. Thus, no containers remain to be contracted under the frame SLA, which in turn means that the specific SLA A.3 leads to a violation of the aggregated SLO Containers.

As part of our ongoing research we are preparing an empirical evaluation of our SLA management approach. This includes more sophisticated examples and use cases, as well as controlled experiments that combine real data from the field with simulation to assess performance, scalability, effectiveness and accuracy of the BizSLAM App.

\subsection{Usefulness}

Having access to the multiple levels of SLA information along the whole supply chain significantly contributes to a better and more efficient planning and execution of transport and logistics services. The data model underlying the BizSLAM App consolidates all information relevant for SLA management of transport and logistics services. Nowadays, such information is scattered across e-mails, spread sheets, and paper documents. Of course, this data model might not cover all cases of SLOs and relationships of the entire transport and logistics industry. However, encouraging feedback from industry partners indicates that the data model covers most of such cases. The organizations we solicited feedback from represented companies of different size (SMEs and large companies) and industry sectors (sea, air, and road carriers, as well as forwarders).

Considering the service level violations in the above scenario, current situation in industry would have seen penalties enforced only long after the logistics service provider suffered the actual losses. This happens because the conformity check in transport and logistics agreements is currently a manual process executed only periodically (e.g., quarterly, half yearly, annually, etc.). Such manual processes might be viable in a small company, but in large companies with high volumes of specific agreements such manual processes become extremely costly. Hence, new online, automated conformity check mechanisms can drastically improve the timeliness of contract violation detection and should thus lead to cost reductions.

\section{Conclusion}

Starting from an identification of industry requirements, this paper presents a runtime SLA management approach for the transport and logistics domain. Specifically, we introduced and demonstrated the usefulness of a novel software component called BizSLAM App that is able to manage SLAs of transport and logistics services at runtime. The App leveraged SLA management approaches from the service-oriented computing field and adapted them to fit the specific requirements of the transports and logistic domain, especially the need to support both frame SLAs and specific SLAs.

The BizSLAM App was developed on top of FIspace.eu, a cloud-based business collaboration platform that offers novel business-to-business collaboration facilities. This 
in turn facilitates applying the BizSLAM App capabilities to other non-computational services, as the platform fosters integrating and combining data, services and Apps of various business stakeholders. As part of the FIspace.eu platform, we are currently adapting the BizSLAM App to the agrifood domain, thereby providing facilities to manage contracts from food production to consumption.

Acknowledgements. We cordially thank our industry partners of the FInest and FIspace projects for their valuable contributions to the SLA data model. In addition, we thank Stephan Heyne for supporting us in implementing the Linked-USDL models, as well as Nadeem Bari for his help in implementing the BizSLAM App. We further express our gratitude to Antonio Manuel Gutierrez, Manuel Resinas and Antonio RuizCortés for earlier collaborations on that subject. Finally, we would like to thank the anonymous reviewers for their constructive comments that benefited this paper.

This work was partially supported by the EU's Seventh Framework Programme (FP7/2007-2013) under grant agreements 285598 (FInest) and 604123 (FIspace).

\section{References}

1. Alliance of European Logistics: A technology roadmap for logistics. Technical Report (October 2013)

2. Andrieux, A., Czajkowski, K., Dan, A., Keahey, K., Ludwig, H., Nakata, T., Pruyne, J., Rofrano, J., Tuecke, S., Xu, M.: Web services agreement specification (WS-Agreement). Specification from the Open Grid Forum (OGF) (March 2007)

3. Augenstein, C., Ludwig, A., Franczyk, B.: Integration of service models - preliminary results for consistent logistics service management. In: Service Research and Innovation Institute Global Conference (SRII 2012), San Jose, Calif., USA (2012)

4. Benaissa, M., Boukachour, J., Benabdelhafid, A.: Web service in integrated logistics information system. In: Int'1 Symposium on Logistics and Industrial Informatics (LINDI 2007). Wildau, Germany (2007)

5. Cuomo, A., Modica, G.D., Distefano, S., Puliafito, A., Rak, M., Tomarchio, O., Venticinque, S., Villano, U.: An SLA-based broker for cloud infrastructures. Journal of Grid Computing 11(1), 1-25 (2013)

6. Dong, H., Hussain, F., Chang, E.: Transport service ontology and its application in the field of semantic search. In: Int'l Conference on Service Operations and Logistics, and Informatics (IEEE/SOLI 2008), vol. 1, pp. 820-824 (October 2008)

7. e-Freight project: D1.3b: e-Freight framework - information models (March 2010), http://www.efreightproject.eu/

8. Feldman, Z., Fournier, F., Franklin, R., Metzger, A.: Proactive event processing in action: a case study on the proactive management of transport processes (industry article). In: 7th Int'l Conference on Distributed Event-based Systems (DEBS 2013), Arlington, Texas, USA (2013)

9. Franklin, R., Metzger, A., Stollberg, M., Engel, Y., Fjørtoft, K., Fleischhauer, R., Marquezan, C., Ramstad, L.S.: Future Internet technology for the future of transport and logistics. In: ServiceWave Conference 2011, Future Internet PPP Track, Ghent, Belgium (2011)

10. Goel, N., Kumar, N., Shyamasundar, R.K.: SLA monitor: A system for dynamic monitoring of adaptive web services. In: 9th European Conference on Web Services (ECOWS 2011), Lugano, Switzerland (2011) 
11. Guihua, N., Fu, M., Xia, H.: A semantic mapping system based on e-commerce logistics ontology. In: World Congress on Software Engineering (WCSE 2009), vol. 2, pp. 133-136 (May 2009)

12. Gutiérrez, A.M., Cassales Marquezan, C., Resinas, M., Metzger, A., Ruiz-Cortés, A., Pohl, K.: Extending WS-Agreement to support automated conformity check on transport and logistics service agreements. In: Basu, S., Pautasso, C., Zhang, L., Fu, X. (eds.) ICSOC 2013. LNCS, vol. 8274, pp. 567-574. Springer, Heidelberg (2013)

13. Hvitved, T., Klaedtke, F., Zalinescu, E.: A trace-based model for multiparty contracts. The Journal of Logic and Algebraic Programming 81(2), 72-98 (2012)

14. Kouki, Y., Ledoux, T.: SLA-driven capacity planning for cloud applications. In: 4th Int'l Conference on Cloud Computing Technology and Science (CloudCom 2012), Taipei, Taiwan, pp. 135-140 (2012)

15. Leitner, P., Ferner, J., Hummer, W., Dustdar, S.: Data-driven and automated prediction of service level agreement violations in service compositions. Distributed and Parallel Databases 31(3), 447-470 (2013)

16. McConnell, A., Parr, G., McClean, S., Morrow, P., Scotney, B.: A SLA-compliant cloud resource allocation framework for n-tier applications. In: 1st Int'l Conference on Cloud Networking (CLOUDNET 2012), Paris, France (2012)

17. Metzger, A., Franklin, R., Engel, Y.: Predictive monitoring of heterogeneous service-oriented business networks: The transport and logistics case. In: Service Research and Innovation Institute Global Conference (SRII 2012), San Jose, Calif., USA (2012)

18. Molina-Jimenez, C., Shrivastava, S., Strano, M.: A model for checking contractual compliance of business interactions. IEEE Trans. on Services Comp. 5(2), 276-289 (2012)

19. Müller, C., Resinas, M., Ruiz-Cortés, A.: Automated analysis of conflicts in WS-Agreement. IEEE Trans. on Services Comp. PP(99), 1 (2013)

20. Munteanu, V., Fortis, T., Negru, V.: An evolutionary approach for SLA-based cloud resource provisioning. In: 27th Int'l Conference on Advanced Information Networking and Applications (AINA 2013), Barcelona, Spain (2013)

21. Papazoglou, M., Pohl, K., Parkin, M., Metzger, M. (eds.): Service Research Challenges and Solutions for the Future Internet: S-Cube - Towards Mechanisms and Methods for Engineering, Managing, and Adapting Service-Based Systems. Springer (2010)

22. Rosario, S., Benveniste, A., Jard, C.: Monitoring probabilistic SLAs in web service orchestrations. In: Int'l Symposium on Integrated Network Management (IM 2009), New York, USA (2009)

23. Verdouw, C., Beulens, A., Wolfert, S.: Towards software mass customization for business collaboration. In: Service Research and Innovation Institute Global Conference (SRII 2014), San Jose, Calif., USA (2014)

24. Wieder, P., Butler, J.M., Theilmann, W., Yahyapour, R. (eds.): Service Level Agreements for Cloud Computing. Springer (2011)

25. Xu, L., Jeusfeld, M.A.: Pro-active monitoring of electronic contracts. In: Eder, J., Missikoff, M. (eds.) CAiSE 2003. LNCS, vol. 2681, pp. 584-600. Springer, Heidelberg (2003)

26. Yahya, B., Mo, J., Bae, H., Lee, H.: Ontology-based process design support tool for vessel clearance system. In: Int'l Conference on Computers and Industrial Engineering (CIE 2010), pp. 1-6 (July 2010)

27. Hua, W.Z., Yousen, H., Yun, D.Z., Wei, Z.: SOA-BPM based information system for promoting agility of third party logistics. In: Int'l Conference on Automation and Logistics (ICAL 2009), Shenyang, China (2009)

28. Zhu, Q., Fung, R.: Design and analysis of optimal incentive contracts between fourth-party and third-party logistics providers. In: Int'l Conference on Automation and Logistics (ICAL 2012), Zhengzhou, China (2012) 